\title{
A systematic review and meta-analysis on the efficacy of Compound Kushen Injection in $\mathbf{3}$ kinds of digestive tract tumor
}

\author{
Wuguang Zhang ${ }^{1}$, Wenqian Gong ${ }^{2}$, Xiansong $\mathrm{He}^{3}$, Changhai $\mathrm{Wu}^{4}$, Xiaolong $\mathrm{Tu}^{5}$ \\ ${ }^{1}$ Oncology Department of Integrated Traditional Chinese and Western Medicine, The Affiliated People's Hospital of Ningbo University, Ningbo, \\ China; ${ }^{2}$ Department of Traditional Chinese Medicine, The Affiliated People's Hospital of Ningbo University, Ningbo, China; ${ }^{3}$ Department of \\ Traditional Chinese Medicine, Ningbo Medical Center Lihuili Hospital, Ningbo, China; ${ }^{4}$ Department of Acupuncture, Ningbo Medical Center \\ Lihuili Hospital, Ningbo, China; ${ }^{5}$ Department of Oncology, Ningbo Hospital of traditional Chinese Medicine, Ningbo, China \\ Contributions: (I) Conception and design: W Gong; (II) Administrative support: W Zhang; (III) Provision of study materials or patients: X He; (IV) \\ Collection and assembly of data: C Wu; (V) Data analysis and interpretation: X Tu; (VI) Manuscript writing: All authors; (VII) Final approval of \\ manuscript: All authors. \\ Correspondence to: Wuguang Zhang. Oncology Department of Integrated Traditional Chinese and Western Medicine, The Affiliated People's Hospital \\ of Ningbo University, Ningbo 315100, China. Email: zhangwuguang91@163.com.
}

Background: Chemotherapy has become the main means to prolong the life of patients with advanced digestive tract cancer; however, it is associated with serious toxicity and side effects. Compound Kushen Injection (CKI) is a pure Chinese herbal preparation, which can assist chemotherapy, inhibit tumor cell proliferation, and reduce adverse reactions of chemotherapy. In this study, we systematically evaluated reports of CKI as an adjuvant to chemotherapeutic treatment of digestive tract cancer in recent years and provided evidence for clinical diagnosis and treatment.

Methods: The databases of PubMed, Chinese Biomedical Literature (CBM), China National Knowledge Infrastructure (CNKI) and Web Of Science were searched for clinical randomized controlled trials (RCTs) related to adjuvant chemotherapy with CKI in the treatment of advanced gastrointestinal tumors published from January 2000 to September 2021. After screening the qualified literatures, RevMan 5.4 software was used to evaluate the bias of the included literatures and perform meta-analysis.

Results: A total of 12 articles were included in the selection, incorporating 1080 study participants in all; meta-analysis results showed that application of the CKI in the process of chemotherapy for digestive tract tumors could improve the efficacy [odds ratio $(\mathrm{OR})=3.11 ; 95 \%$ confidence interval (CI): 2.26 to 4.47 , $\mathrm{Z}=7.00, \mathrm{P}<0.00001$ ], increase the patients' median survival time (months) (OR =3.00; 95\% CI: 1.47 to 4.52 , $\mathrm{Z}=3.84, \mathrm{P}=0.0001)$, increase the level of $\mathrm{CD}^{+}$[mean difference (MD) $=4.11 ; 95 \% \mathrm{CI}: 3.24$ to 4.98], CD4 level (MD $=8.24 ; 95 \% \mathrm{CI}: 3.72$ to 12.76$)$, reduce the $\mathrm{CD}^{+}$level ( $\mathrm{MD}=-5.42 ; 95 \% \mathrm{CI}:-8.09$ to -2.76$)$, reduce the tumor markers carcinoembryonic antigen (CEA; $\mathrm{MD}=-14.26 ; 95 \% \mathrm{CI}:-14.81$ to -13.71 ), CA199 (MD =-138.87; 95\% CI: -143.21 to -132.52 ), and reduce the adverse reactions of chemotherapy: leukopenia (OR $=0.28 ; 95 \%$ CI: 0.19 to 0.43$)$, thrombocytopenia (OR $=0.38 ; 95 \%$ CI: 0.24 to 061$)$, decreased hemoglobin (OR $=0.55$; $95 \%$ CI: 0.31 to 0.98$)$, and nausea and vomiting symptoms (OR =0.35; $95 \%$ CI: 0.24 to 0.53$)$.

Discussion: Adjuvant chemotherapy with CKI in the treatment of digestive tract tumors can effectively improve the symptoms of patients, improve immunity, reduce the level of serum tumor markers, improve efficacy, and reduce toxic and side effects.

Keywords: Compound Kushen Injection (CKI); digestive tract cancer; chemotherapy; efficacy; meta-analysis

Submitted Nov 03, 2021. Accepted for publication Dec 14, 2021.

doi: 10.21037/jgo-21-774

View this article at: https://dx.doi.org/10.21037/jgo-21-774

(C) Journal of Gastrointestinal Oncology. All rights reserved. 


\section{Introduction}

Gastric cancer, esophageal cancer, and intestinal cancer are the diseases with the highest incidence and the highest mortality among all malignant tumors of the digestive tract (1). Digestive tract tumors have occult characteristics and are not obvious in the early stage. At the time of diagnosis, most patients are in the middle and advanced stage and have exceeded the optimal surgical window of opportunity. Chemotherapy has become the main means to prolong the patient's life (2). Although chemotherapy can kill cancer cells and control clinical symptoms, it also destroys normal cells and tissues and brings toxic side effects, and some patients have to cease treatment due to inability to tolerate the side effects of chemotherapy (3). Therefore, the medical community attaches great importance to studying how to reduce the adverse reactions caused by chemotherapy and improve the efficacy through adjuvant means. With the continuous in-depth study of traditional Chinese medicine (TCM) in this field, more and more researchers have found that the treatment of integrated TCM and western medicine has certain advantages in improving the quality of life of patients with advanced digestive tract cancer and reducing the pain caused by cancer (4). Compound Kushen Injection (CKI) is a pure Chinese herbal preparation extracted from Radix Sophorae Flavescentis (Ku Shen) and Poria cocos (Fu Ling). Studies have shown that its application in antitumor treatment can inhibit tumor cell proliferation, reduce adverse reactions of chemotherapy, and improve patient tolerance (5). A study has revealed that the potential molecular mechanism of CKI can regulate PI3K/AKT and Toll-like receptor signaling pathways by interfering with hub genes such as AKR1B1, MMP2 and PTGERR3, so as to inhibit cancer cell proliferation and regulate immunity (6). Previously, researchers have conducted systematic evaluations of CKI in non-small cell lung cancer (7), breast cancer (8), but there is no comprehensive systematic evaluation for three common digestive tract tumors: gastric cancer, esophageal cancer, and intestinal cancer. In this study, we searched for relevant clinical controlled trials to objectively evaluate the short-term efficacy and safety of CKI combined with chemotherapy in the treatment of patients with advanced gastric cancer, esophageal cancer, and intestinal cancer in order to provide a basis for clinical medication. We present the following article in accordance with the PRISMA reporting checklist (available at https://dx.doi.org/10.21037/ jgo-21-774).

\section{Methods}

\section{Literature inclusion criteria}

(I) Study types: all included studies were randomized controlled trials (RCTs), whether there was only 1 center in the study was not limited, allocation concealment method and blind method of the study were not limited, and the languages of the articles were Chinese and English; (II) study participants: all participants were human, and the studies of rats, rabbits, monkeys, dogs, and other animals were excluded. All the patients were diagnosed with gastrointestinal tumors (only colon cancer, gastric cancer, esophageal cancer), and confirmed by histopathology and cytology. The survival time of the participants was more than 3 months, regardless of age, ethnicity, and cancer stage, and the patients had no contraindications to chemotherapy; (III) intervention measures: all the studies were divided into the control group and the experimental group for intervention. Both participant groups underwent chemotherapy, with the addition of CKI in the experimental group (intravenous drip); (IV) outcome indicators: (i) short-term efficacy. Efficacy after 2-3 courses of treatment, efficacy including partial relief (PR) and complete relief (CR), effective rate $=(C R+P R) /$ total number of cases; (ii) long-term efficacy. The median survival time of the patients; (iii) immune indicators: including $\mathrm{CD}^{+}, \mathrm{CD}^{+}$, and $\mathrm{CD} 8^{+}$ values in serum after treatment (\%); (iv) tumor marker indicators: serum carcinoembryonic antigen (CEA), serum carbohydrate antigen 19-9 (CA199); (v) adverse reactions: including the number and proportion of cases of leukopenia, thrombocytopenia, hemoglobin reduction, nausea and vomiting after treatment; $(\mathrm{V})$ observation time: 2 courses of treatment ( 1 or 2 months) as the observation time point.

\section{Literature exclusion criteria}

(I) Reviews, experience sharing, and case analysis. Such articles were excluded because there was no specific data. Controlled studies and observational studies were excluded because there was no control method; (II) cancers other than colon cancer, gastric cancer, and esophageal cancer, such as lung cancer, breast cancer; (III) complex intervention measures, combination with other TCM treatment options (such as herbal decoction, massage, acupuncture). 


\section{Literature search}

(I) Search strategy: The keywords ("compound kushen injection" or "kushen injection" or "matrine injection") AND ("gastric cancer" or "Esophageal cancer" or "colorectal cancer") were searched; (II) database: PubMed, Chinese Biomedical Literature (CBM), China National Knowledge Infrastructure (CNKI), Web of Science; (III) filter setting: the time of literature publication (January 2000-September 2021), literature type (RCT).

\section{Selection of literatures}

After two researchers had independently completed the retrieval, the literature data were entered into EndNote X9 software (Clarivate, Philadelphia, PA, USA) for subsequent management. Using the de-duplication function of the software, the repeated documents were eliminated, and the title, abstract, and full text of the literatures were read. The unqualified articles were excluded. In case of any dispute in this process, a 3 rd person could intervene and coordinate after discussion.

\section{Data extraction and conversion}

After the literature screening, two researchers-reread the full texts of articles, extracting literature feature information (author, publication time, study site), participant information (gender, age, disease type, tumor stage), information of intervention indicators (number of participants in groups, intervention methods) and outcome information (outcome indicators). In the case of missing data, the article was excluded.

\section{Literature risk of bias assessment}

The risk of bias assessment module of RevMan 5.4 software (The Cochrane Collaboration, Copenhagen, Denmark, 2020) was used to assess the 3 evaluation indicators: (I) random allocation method; (II) blind method; (III) implementation of allocation concealment; (IV) data integrity; (V) selective reporting bias; (VI) other biases, including "low risk", "unclear", and "high risk".

\section{Statistical methods}

(I) Analysis was performed using RevMan 5.4 software; (II) standardized mean difference (SMD), effect size, and
$95 \%$ confidence interval (CI) were used for continuous indicators, and odds ratio (OR) effect size, and $95 \%$ CI were used for discrete indicators (binary classification), with $\mathrm{P}<0.05$ indicating statistical significance; (III) mantelHaenszel model was used for each outcome indicator (short-term efficacy, immune indicators, tumor marker indicators, adverse reactions); (IV) forest plot was used to display the effect size; (V) $\mathrm{I}^{2}$ analysis and $\mathrm{Q}$ were used to verify the heterogeneity of literatures. When $\mathrm{I}^{2}>50 \%$ or $\mathrm{P}<0.1$ heterogeneity was indicated, and the random effects model was used, otherwise, the fixed effects model was used. Mantel-Haenszel model was used for OR effect size, and inverse variance model was used for SMD effect size; (VI) if heterogeneity was suggested between studies and the source of heterogeneity could not be determined, only descriptive analysis was done; (VII) sensitivity analysis was performed on the results; (VIII) publication bias was represented using funnel plots.

\section{Results}

\section{Literature screening process and results}

The literature retrieval flow chart is shown in Figure 1. Initially, 523 articles were retrieved, and after screening, 12 articles (9-20) were included for selection, with a total of 1,080 patients included.

\section{Basic characteristics of studies}

The basic characteristics, intervention indicators, observation time, and outcome indicators of the included articles are shown in Table 1. The youngest participant was 28 years old and the oldest was 82 years old. The minimum number of cases in the grouping was 30 and the maximum was 80 , including 5 patients with colorectal cancer, 4 patients with gastric cancer, and 3 patients with esophageal cancer.

\section{Literature bias assessment}

In this study, with the exception of article (18), all other studies indicated the use of randomization method, so there maybe selection bias caused by randomization method; no studies indicated whether allocation concealment was used, so there might have been selection bias caused by allocation concealment; all articles did not describe blind method, which might have caused operational bias in the analysis; all articles did not record data drop-out cases in detail, which 


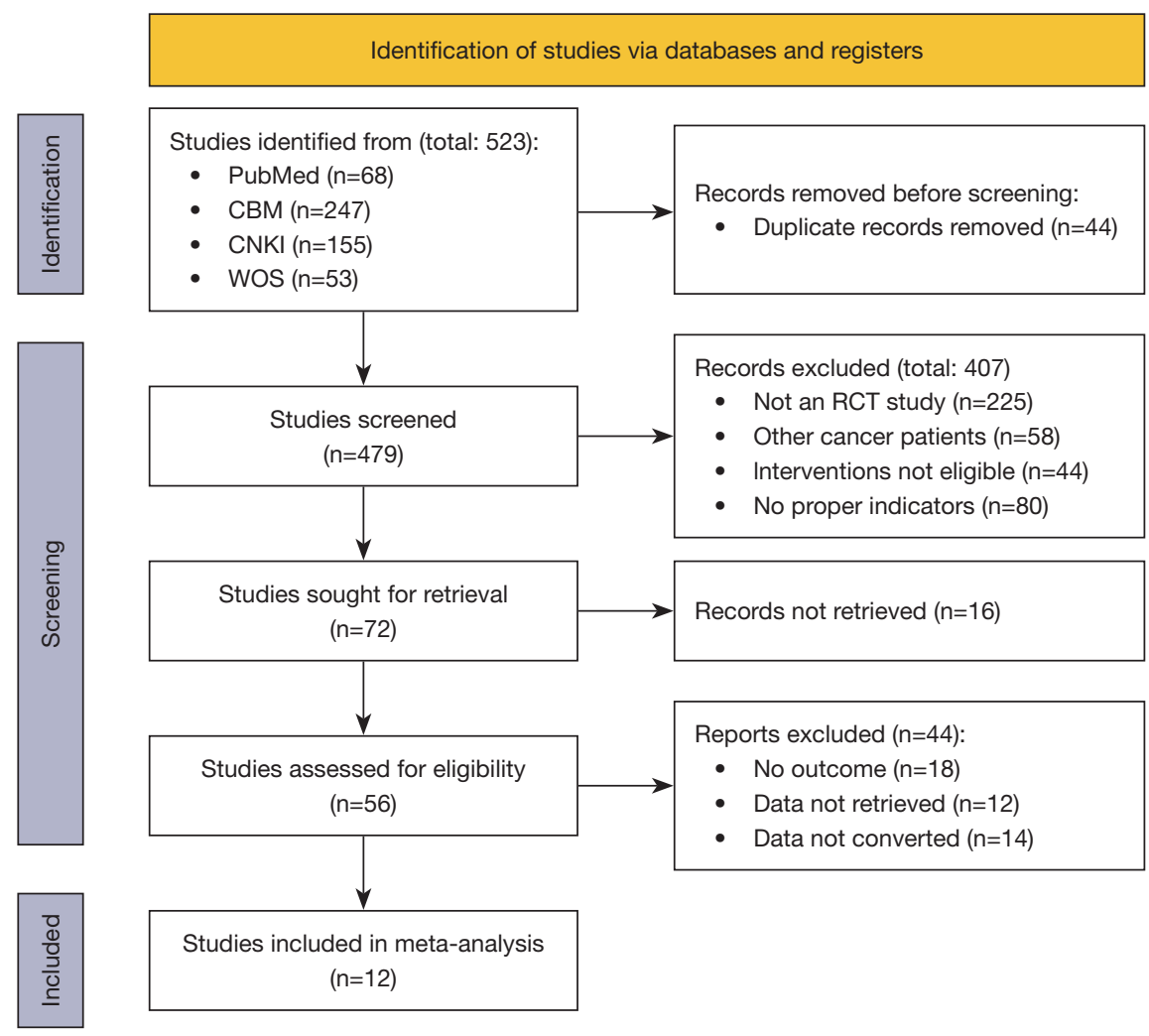

Figure 1 Literature selection flow chart. RT, randomized controlled trial; CBM, Chinese Biomedical Literature Database; CKNI, China National Knowledge Infrastructure; WOS, Web of Science.

might have caused some attribution bias; selective reporting and other bias were not observed (Figures 2,3).

\section{Meta-analysis results}

\section{Short-term efficacy}

With the exception of article (9), all studies reported the effect of CKI on patients with digestive tract cancer undergoing chemotherapy. A total of 510 patients were included in the experimental group and the control group, without statistical heterogeneity between the studies $\left(\mathrm{I}^{2}=0 \%\right.$, $\mathrm{P}=0.98)$. The fixed effects model analysis was used to obtain the pooled size (OR $=3.11$; 95\% CI: 2.26 to 4.27), which indicated that the application of CKI as an adjuvant to digestive tract cancer chemotherapy can improve the efficacy $(Z=7.00 ; P<0.00001)$. The patients were further divided into a colorectal cancer subgroup $(\mathrm{OR}=2.67 ; 95 \%$ CI: 1.59 to 4.50$)$, gastric cancer subgroup (OR $=3.39 ; 95 \%$ CI: 2.09 to 5.49 ), and esophageal cancer subgroup (OR $=3.41 ; 95 \%$ CI: 1.67 to 6.96 ) according to cancer type.
There was no statistical heterogeneity among the three internal subgroups (Figure 4).

\section{Long-term efficacy}

Four studies $(9,18-20)$ reported the median survival time (months) of CKI on patients with digestive tract cancer undergoing chemotherapy. A total of 202 patients were included in the experimental group and the control group respectively, with statistically significant heterogeneity between the studies $\left(\mathrm{I}^{2}=66 \%, \mathrm{P}=0.03\right)$. The random effects model analysis was used to obtain the pooled size (OR $=3.00 ; 95 \%$ CI: 1.47 to 4.52 ), which indicated that the application of CKI as an adjuvant to digestive tract cancer chemotherapy can improve the median survival time ( $\mathrm{Z}=3.84 ; \mathrm{P}=0.0001$ ) (Figure 5).

\section{Immune function indicators}

The application of CKI in the chemotherapy of digestive tract tumors can increase the levels of $\mathrm{CD}^{+}$and $\mathrm{CD} 4^{+}$and reduce the level of $\mathrm{CD}^{+}$(Table 2). 


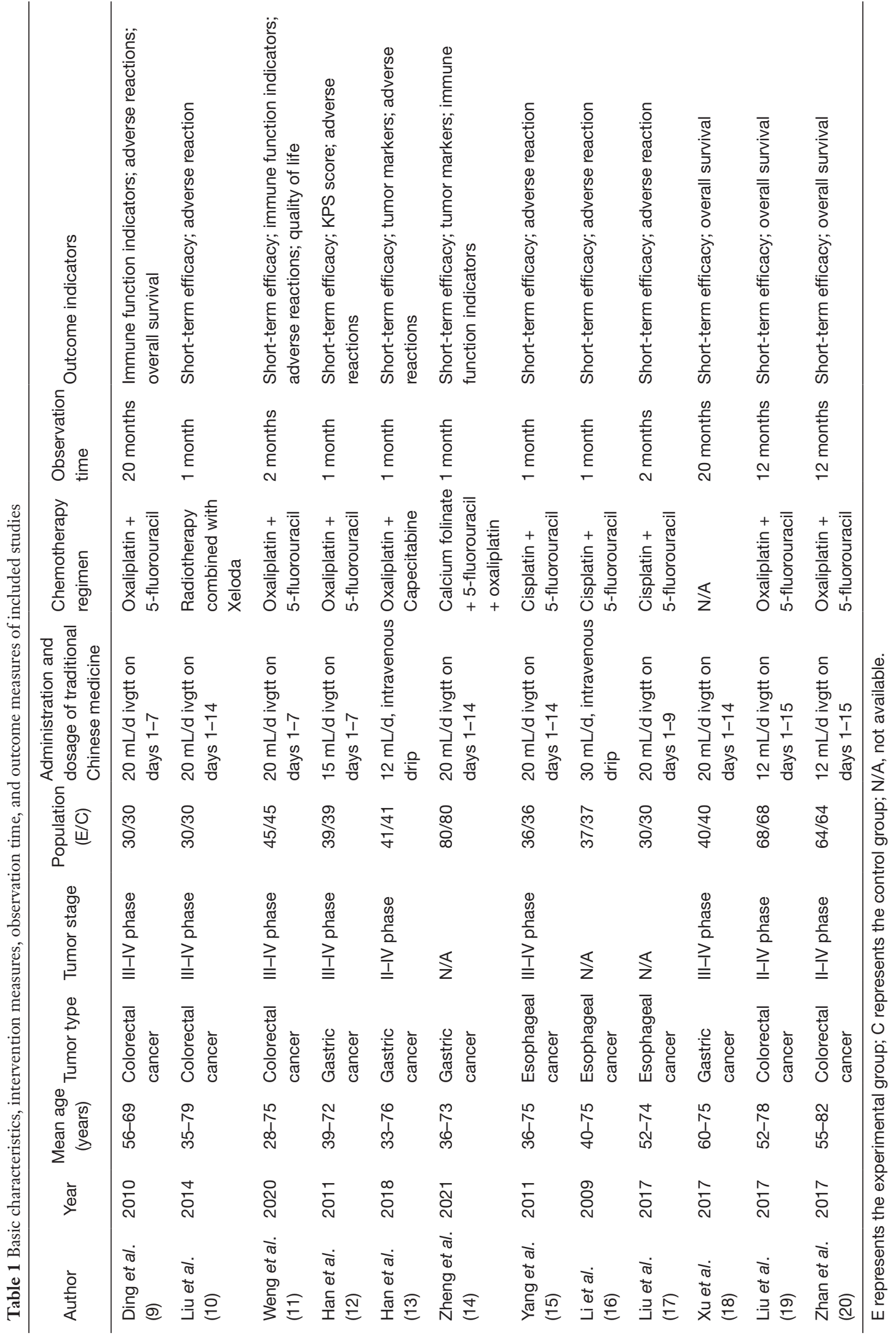




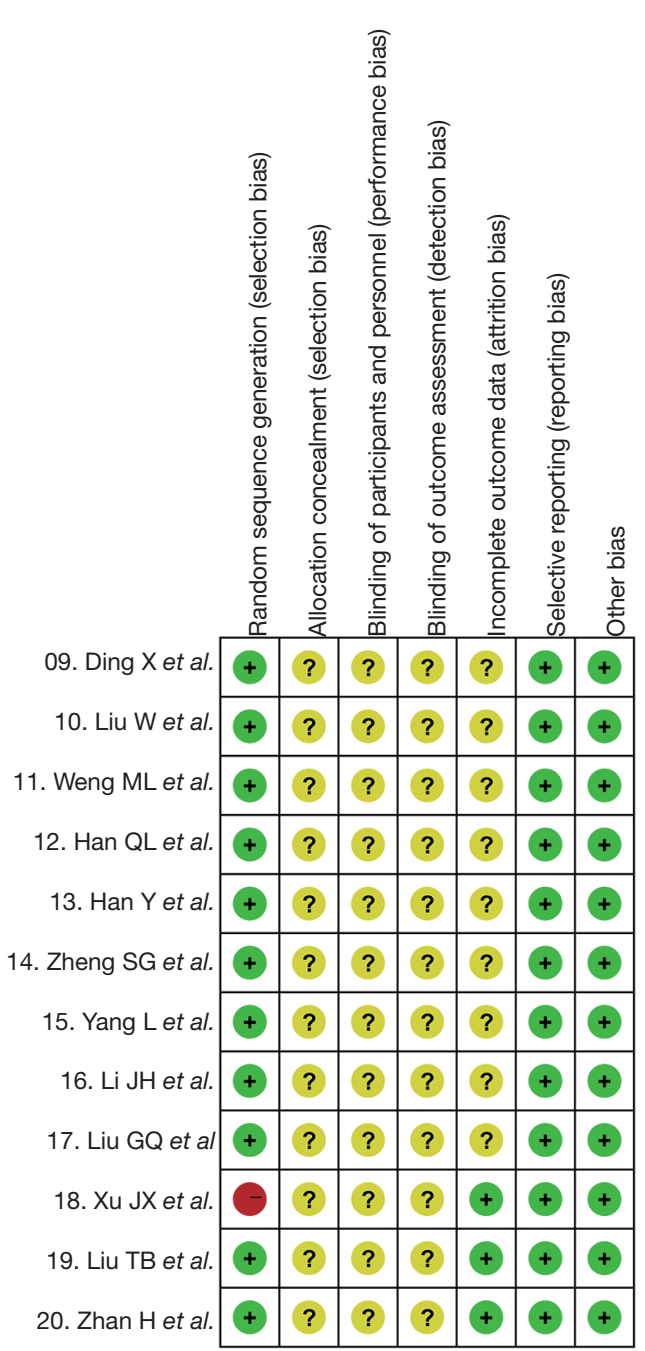

Figure 2 Risk of bias summary (9-20).

\section{Tumor marker indicators}

As shown in Table 3, CKI can reduce the levels of tumor markers CEA and CA199 during chemotherapy for digestive tract tumors.

\section{Adverse reactions}

Participants who received CKI during the course of chemotherapy for digestive tract tumors experienced lower rates of adverse reactions of leukopenia, thrombocytopenia, decreased hemoglobin, nausea and vomiting symptoms than those in the control group (Table 4).

\section{Heterogeneity investigation}

In the meta-analysis of immune function indicators, there was statistically significant heterogeneity between the studies, and the source of heterogeneity may have been related to different tumor types of patients and different nodes of observation time.

\section{Sensitivity analysis}

In the analysis of short-term efficacy, if the random effect model was used for analysis, the pooled effect size (OR $=3.20$; $95 \%$ CI: 2.19 to 4.67 ) was obtained, which was not significantly different from the results of the fixed effect model analysis, so the results could be considered stable.

\section{Publication bias analysis}

In the analysis of the short-term efficacy, 8 included studies were evenly distributed on the left and right sides in the funnel plot, suggesting that the possibility of publication bias was small, as shown in Figure 6.

\section{Discussion}

For patients with advanced gastrointestinal malignancies who have missed the optimal period of surgical treatment, chemoradiotherapy and molecular targeted therapy are the only existing treatment methods, but molecular targeted drug therapy is expensive and the efficacy varies according to individual patients, and chemoradiotherapy can bring serious toxicity, which limits the available treatment methods to some extent (21). In recent years, the adjuvant therapy of TCM has attracted increasing attention in the field of cancer treatment, and it has obvious advantages in relieving the toxic side effects and improving the efficacy of chemotherapy (22). The CKI is refined and processed from two traditional Chinese herbal medicines, Radix Sophorae Flavescentis and Poria cocos, and its main biological components are alkaloids such as matrine, oxymatrine, and Sophora flavescens, which can inhibit tumor cell proliferation, induce tumor cell apoptosis, control tumor cell infiltration and metastasis, and enhance the sensitivity of chemotherapeutic drugs, thus playing an anti-tumor role (23). In addition, matrine and oxymatrine also have analgesic, anti-infective, hemostatic, and immuneenhancing effects (24).

In this study, 9 clinical RCTs of CKI in the adjuvant treatment of digestive tract tumors were included, including 3 studies of colorectal cancer, 3 studies of gastric cancer, and 3 studies of esophageal cancer. Meta-analysis results showed that CKI had obvious advantages in the adjuvant process of chemotherapy, which were reflected as follows: (I) it could enhance the short-term efficacy of cancer treatment 


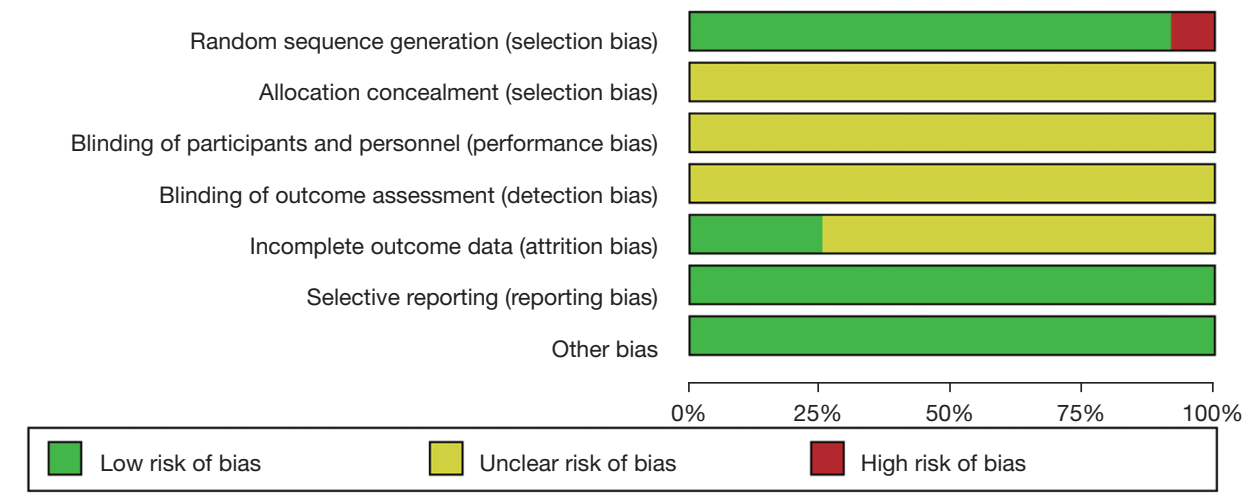

Figure 3 Risk of bias graph.

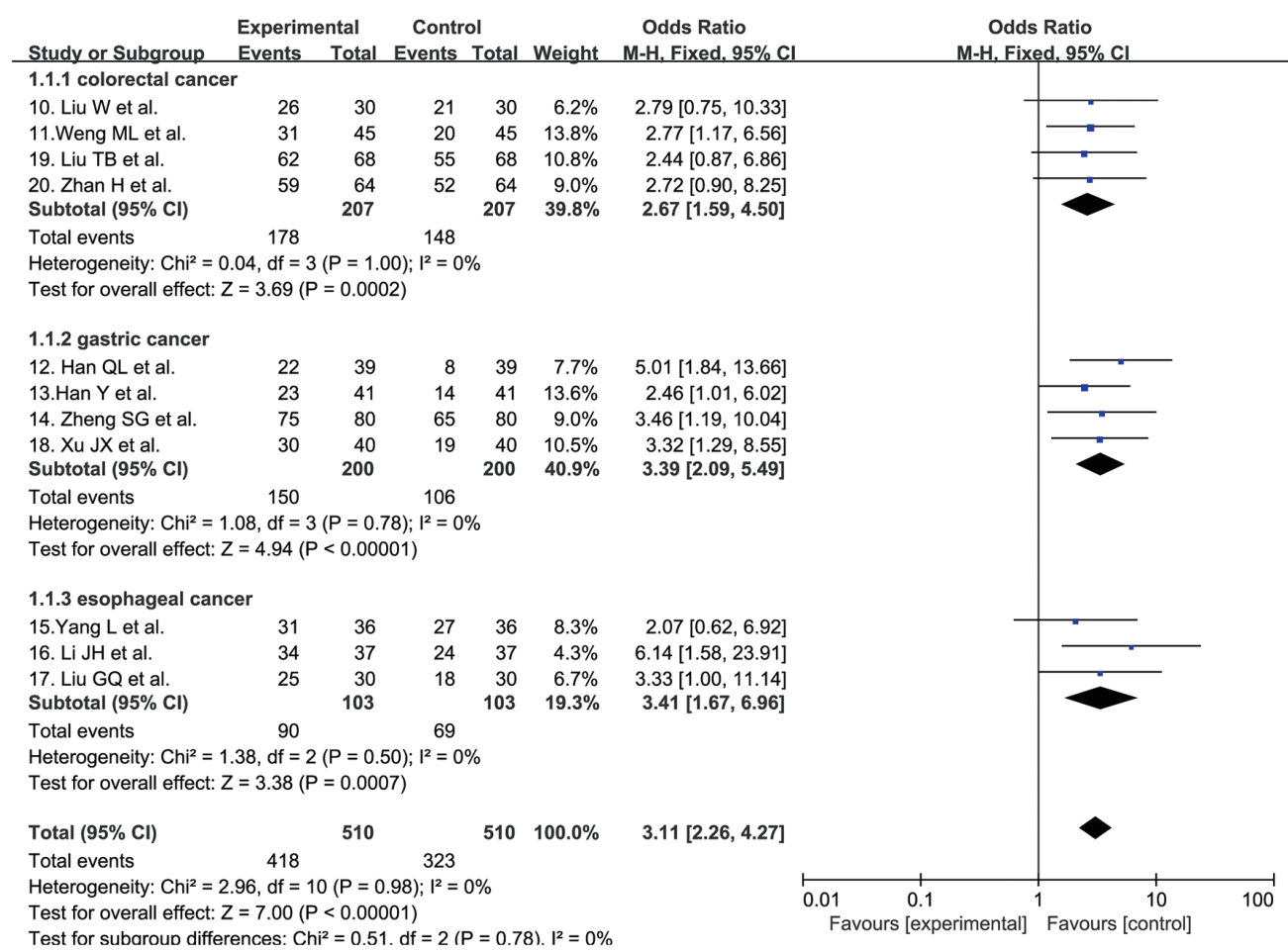

Figure 4 Combined analysis of the effect of CKI on the short-term efficacy of digestive tract tumors. CKI, compound Kushen injection; CI, confidence interval.

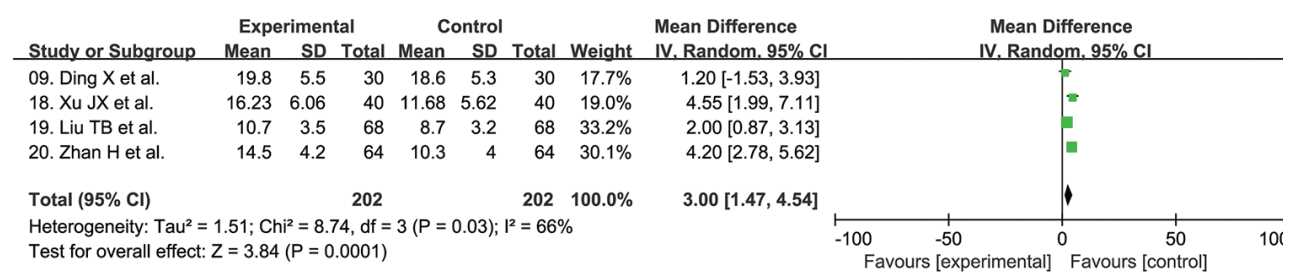

Figure 5 Combined analysis of the effect of CKI on the median survival time of digestive tract tumors. CKI, compound Kushen injection; CI, confidence interval. 
Table 2 Meta-analysis results of immune indicators

\begin{tabular}{lcccccc}
\hline Factors & Number of participants & Number of studies & Statistical method & I $^{2} \mathrm{C}$ with P value & Effect estimate & $P$ value \\
\hline $\mathrm{CD}^{+}$ & 310 & 3 & MD (IV, fixed, 95\% Cl) & 0\% with 0.56 & $4.11(3.24,4.98)$ & $<0.00001$ \\
$\mathrm{CD}^{+}$ & 310 & 3 & MD (IV, random, 95\% Cl) & $96 \%$ with 0.00001 & $8.24(3.72,12.76)$ & 0.0003 \\
$\mathrm{CD}^{+}$ & 220 & 3 & MD (IV, random, 95\% Cl) & $88 \%$ with 0.004 & $-5.42(-8.09,-2.76)$ & $<0.00001$ \\
\hline
\end{tabular}

MD, mean difference; $95 \% \mathrm{Cl}, 95 \%$ confidence interval.

Table 3 Meta-analysis results of tumor marker indicators

\begin{tabular}{|c|c|c|c|c|c|c|}
\hline Factors & $\begin{array}{c}\text { Number of participating } \\
\text { patients }\end{array}$ & $\begin{array}{l}\text { Number of } \\
\text { literatures }\end{array}$ & Statistical method & $\mathrm{I}^{2} \mathrm{C}$ with $\mathrm{P}$ value & Effect estimate & $P$ value \\
\hline CEA & 242 & 2 & MD (IV, fixed, 95\% Cl) & $0 \%$ with 0.86 & $-14.26(-14.81,-13.71)$ & $<0.00001$ \\
\hline
\end{tabular}

CEA, carcinoembryonic antigen; CA199, carbohydrate antigen 19-9; MD, mean difference; 95\% Cl, 95\% confidence interval.

Table 4 Meta-analysis results of adverse reactions

\begin{tabular}{lcccccc}
\hline Factors & $\begin{array}{c}\text { Number of } \\
\text { participants }\end{array}$ & Number of studies & Statistical method & I $^{2} \mathrm{C}$ with P value & Effect estimate & $P$ value \\
\hline Leukopenia & 576 & 8 & OR $(\mathrm{M}-\mathrm{H}$, fixed, $95 \% \mathrm{Cl})$ & $0 \%$ with 0.83 & $0.28(0.19,0.43)$ & $<0.00001$ \\
Thrombocytopenia & 516 & 7 & OR $(\mathrm{M}-\mathrm{H}$, fixed, $95 \% \mathrm{Cl})$ & $0 \%$ with 0.81 & $0.38(0.24,0.61)$ & $<0.00001$ \\
Hemoglobin decreased & 232 & 3 & OR $(\mathrm{M}-\mathrm{H}$, fixed, 95\% Cl) & $0 \%$ with 0.58 & $0.55(0.31,0.98)$ & 0.04 \\
Nausea and vomiting & 576 & 8 & OR $(\mathrm{M}-\mathrm{H}$, fixed, $95 \% \mathrm{Cl})$ & $0 \%$ with 0.68 & $0.35(0.24,0.53)$ & $<0.00001$ \\
\hline
\end{tabular}

OR, odds ratio; $\mathrm{M}-\mathrm{H}$, Mantel-Haenszel; 95\% Cl, 95\% confidence interval.

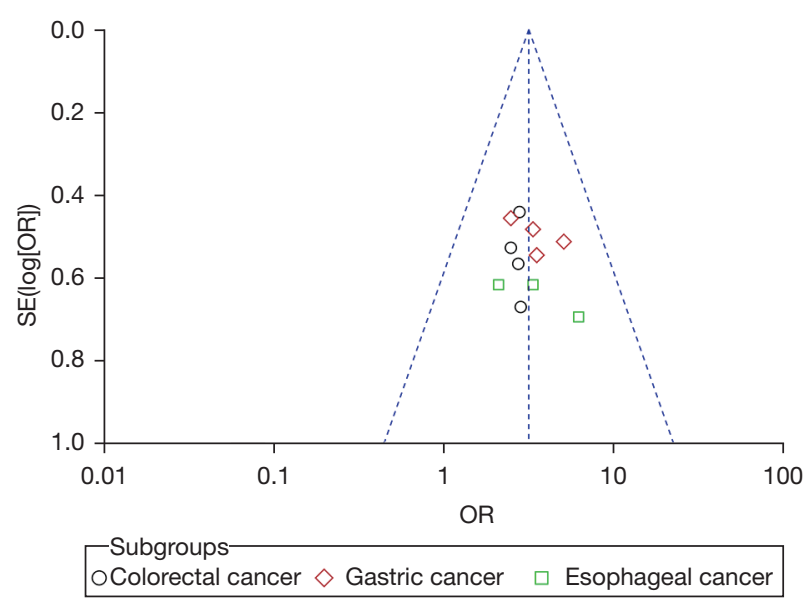

Figure 6 Funnel plot.

and significantly improve the patient's symptoms; (II) it could enhance physical immunity and improve the patient's chemotherapy tolerance; (III) it could significantly reduce the level of serum tumor markers and improve the effect of chemotherapy; (IV) it could reduce the incidence of adverse reactions and reduce the toxic and side effects caused by chemotherapy, such as leukopenia, thrombocytopenia, hemoglobin decreased, nausea and vomiting and other gastrointestinal reactions, liver function injury, renal impairment, and peripheral neurotoxicity.

In literature (18), CKI combined with anticancer drug teggio (s-1) were used in the treatment of advanced gastric cancer patients. The efficacy was better comparing to the control group treated with anticancer drug (s-1) alone, S-1 was a third-generation fluorouracil anticancer drug, which could interfere with the synthesis of RNA in tumor cells and inhibit their growth. The CKI can inhibit the protein phosphorylation level, interfere with the protein expression of tumor cells, and finally inhibit the proliferation of tumor cells, also it can inhibit the growth of tumor blood vessels by inhibiting the transcription of vascular endothelial growth factor gene (25). Together, 
they played an anti-cancer gain effect.

Our results show that CKI can reduce the levels of serum tumor markers CEA and CA199 during the chemotherapy of gastrointestinal malignant tumors. Modern pharmacological studies show that matrine contained in CKI can induce apoptosis of solid tumor cells and prevent DNA biosynthesis of tumor cells. It can promote normal differentiation and programmed apoptosis of tumor cells without destroying normal cells, so as to reduce the level of serum tumor markers (26).

In the study by Zhang et al. (27), 29 controlled clinical trials were included to study the efficacy of adjuvant chemotherapy with CKI in the treatment of esophageal cancer, and it was found that compared with chemotherapy alone, CKI combined with chemotherapy could improve the efficacy of esophageal cancer treatment and reduce adverse reactions. This study expanded the scope of the study and incorporated participants in studies of the three most common gastrointestinal tumors. The results showed that adjuvant chemotherapy with CKI could significantly improve the efficacy, improve immunity, and reduce toxicity and side effects, which was consistent with the results of the above study. In our study, the participants were divided into three subgroups (colorectal cancer, gastric cancer, esophageal cancer), and the results showed that CKI had a significant effect on all three malignant tumors. The short-term efficacy of gastric cancer and esophageal cancer was higher than that of chemotherapy alone $(\mathrm{OR}=3.41)$, which was higher than that of colorectal cancer $(\mathrm{OR}=2.77)$. However, there was no sufficient evidence whether the efficacy of CKI for gastric cancer and esophageal cancer was higher than that of colorectal cancer.

This study still had some limitations, as follows: (I) the number of included literatures was small, the number of participants was still small, and there was a lack of multicenter, large-sample size RCTs; (II) the quality of included studies was not high, 1 article didn't use randomization method for grouping, 9 included articles did not describe the allocation concealment, did not describe the blind method, did not count the dropout cases, there may have been a certain bias, but the quality was generally good; (III) important long-term efficacy indicators, such as patient quality of life after treatment, 3-year mortality, and 5-year mortality were not reported in the studies, therefore, the results of this study are limited to the short-term efficacy.

\section{Conclusions}

Adjuvant chemotherapy with CKI in the treatment of gastrointestinal cancer can effectively improve the symptoms of patients, improve immunity, reduce the level of serum tumor markers, improve efficacy, and reduce toxicity and side effects; however, more high-quality, multicenter, large-sample RCTs need to be included in clinical practice to provide stronger evidence.

\section{Acknowledgments}

Funding: None.

\section{Footnote}

Reporting Checklist: The authors have completed the PRISMA reporting checklist. Available at https://dx.doi. org/10.21037/jgo-21-774

Conflicts of Interest: All authors have completed the ICMJE uniform disclosure form (available at https://dx.doi. org/10.21037/jgo-21-774). The authors have no conflicts of interest to declare.

Ethical Statement: The authors are accountable for all aspects of the work in ensuring that questions related to the accuracy or integrity of any part of the work are appropriately investigated and resolved.

Open Access Statement: This is an Open Access article distributed in accordance with the Creative Commons Attribution-NonCommercial-NoDerivs 4.0 International License (CC BY-NC-ND 4.0), which permits the noncommercial replication and distribution of the article with the strict proviso that no changes or edits are made and the original work is properly cited (including links to both the formal publication through the relevant DOI and the license). See: https://creativecommons.org/licenses/by-nc-nd/4.0/.

\section{References}

1. Wang JC, Tian JH, Ge L, et al. Which is the best Chinese herb injection based on the FOLFOX regimen for gastric cancer? A network meta- analysis of randomized controlled trials. Asian Pac J Cancer Prev 2014;15:4795-800.

2. Sun Z, Jia J, Du F, et al. Clinical significance of serum 
tumor markers for advanced gastric cancer with the firstline chemotherapy. Transl Cancer Res 2019;8:2680-90.

3. Wu R, Shen Z, Yu N, et al. Efficacy and safety of liposome-paclitaxel/liposome-paclitaxel combined with S-1 in 17 advanced gastric cancer patients with poor performance status. Transl Cancer Res 2019;8:1690-8.

4. Lu Y, Li CS, Dong Q. Chinese herb related molecules of cancer-cell-apoptosis: a minireview of progress between Kanglaite injection and related genes. J Exp Clin Cancer Res 2008;27:31.

5. Guo YM, Huang YX, Shen HH, et al. Efficacy of Compound Kushen Injection in Relieving Cancer-Related Pain: A Systematic Review and Meta-Analysis. Evid Based Complement Alternat Med 2015;2015:840742.

6. Zhou W, Wu C, Zhao C, et al. An Advanced Systems Pharmacology Strategy Reveals AKR1B1, MMP2, PTGER3 as Key Genes in the Competing Endogenous RNA Network of Compound Kushen Injection Treating Gastric Carcinoma by Integrated Bioinformatics and Experimental Verification. Front Cell Dev Biol 2021;9:742421.

7. Wu X, Chung VCH, Lu P, et al. Chinese Herbal Medicine for Improving Quality of Life Among Nonsmall Cell Lung Cancer Patients: Overview of Systematic Reviews and Network Meta-Analysis. Medicine (Baltimore) 2016;95:e2410.

8. Zhang $\mathrm{D}, \mathrm{Wu} \mathrm{J}$, Wang $\mathrm{K}$, et al. Which are the best Chinese herbal injections combined with XELOX regimen for gastric cancer?: A PRISMA-compliant network metaanalysis. Medicine (Baltimore) 2018;97:e0127.

9. Ding X, Xiao XY, Yang XY, et al. Clinical observation of compound Sophora flavescens injection combined with FOLFOX4 regimen in the treatment of advanced colorectal cancer. Chinese Journal of Cancer 2010;20:860-3.

10. Liu W, Cheng XL, Dong F, et al. Clinical study of compound Sophora flavescens injection combined with concurrent chemoradiotherapy in the treatment of locally advanced rectal cancer. Tianjin Pharmacy 2014;26:33-6.

11. Weng ML, Zheng QH, Chen JX. Effect of compound Sophora flavescens injection combined with oxaliplatin containing chemotherapy on short-term efficacy and immune cellular function in patients with advanced colon cancer. Zhejiang Journal of Traditional Chinese Medicine 2020;5 5:207-8.

12. Han QL, Zhang LF, Li J, et al. Observation on the efficacy of compound Sophora flavescens injection combined with chemotherapy in the treatment of gastric cancer. China
Medical Herald 2011;8:68-9.

13. Han Y. Clinical efficacy of compound Sophora flavescens injection combined with chemotherapy in the treatment of gastric cancer. Journal of Clinical Rational Drug Use 2018;11:60-1.

14. Zheng SG, Yang XK, Xie HM. Effects of compound Sophora flavescens injection on immune function and serum CEA, CA724 and CA199 levels in patients with gastrointestinal malignancies. Jilin Medical Science 2021;42:2196-8.

15. Yang L, Fu B. Clinical observation of compound Sophora flavescens combined injection with concurrent chemoradiotherapy in the treatment of advanced esophageal cancer. Evaluation and Analysis of Drug Use in Chinese Hospitals 2011;11:168-9.

16. Li JH, Chen X, Li Q. Compound Sophora flavescens injection combined with radiotherapy and $\mathrm{FP}$ regimen in the treatment of middle and late stage upper esophageal cancer. Pharmaceutical Progress 2009;33:325-7.

17. Liu GQ, Zhao H. Observation on the efficacy of combined chemotherapy with compound Sophora flavescens injection in the treatment of advanced esophageal cancer. Journal of Practical Traditional Chinese Medicine 2017;33:535-6.

18. Xu JX, Zhang MJ, Wang AR. Short term efficacy and survival of compound Sophora flavescens injection combined with teggio (s-1) in the treatment of middle and advanced gastric cancer in the elderly. Shaanxi Traditional Chinese Medicine 2017;38:1505-6.

19. Liu TB, Ning ZC, Zhang C. Effect of compound Sophora flavescens injection on Th17 cells and IL-17 factor in patients with rectal cancer and its long-term clinical efficacy. Chinese Journal of Integrated Traditional and Western Medicine Digestion 2017;25:108-11.

20. Zhan H, Zhang HX. Effects of compound Sophora flavescens injection combined with FOLFOX4 chemotherapy on adjuvant T cells, IL-17 factor and survival rate in patients with rectal cancer. International Journal of Traditional Chinese Medicine 2017;39:412-5.

21. He R, Ou S, Chen S, et al. Network Pharmacology-Based Study on the Molecular Biological Mechanism of Action for Compound Kushen Injection in Anti-Cancer Effect. Med Sci Monit 2020;26:e918520.

22. Wang H, Hu H, Rong H, et al. Effects of compound Kushen injection on pathology and angiogenesis of tumor tissues. Oncol Lett 2019;17:2278-82.

23. Jin Y, Yang Q, Liang L, et al. Compound kushen injection suppresses human acute myeloid leukaemia by regulating the Prdxs/ROS/Trx1 signalling pathway. J Exp Clin 
Cancer Res 2018;37:277.

24. Yang Y, Sun M, Yao W, et al. Compound kushen injection relieves tumor-associated macrophage-mediated immunosuppression through TNFR1 and sensitizes hepatocellular carcinoma to sorafenib. J Immunother Cancer 2020;8:e00317.

25. Liu S, Hu X, Fan X, et al. A Bioinformatics Research on Novel Mechanism of Compound Kushen Injection for Treating Breast Cancer by Network Pharmacology and Molecular Docking Verification. Evid Based Complement Alternat Med 2020;2020:2758640.

Cite this article as: Zhang $\mathrm{W}$, Gong $\mathrm{W}, \mathrm{He} \mathrm{X}, \mathrm{Wu} \mathrm{C}$, Tu X. A systematic review and meta-analysis on the efficacy of Compound Kushen Injection in 3 kinds of digestive tract tumor. J Gastrointest Oncol 2021;12(6):2919-2929. doi: 10.21037/jgo-21774
26. Zhou $\mathrm{W}, \mathrm{Wu}$ J, Zhang J, et al. Integrated bioinformatics analysis to decipher molecular mechanism of compound Kushen injection for esophageal cancer by combining WGCNA with network pharmacology. Sci Rep 2020;10:12745.

27. Zhang D, Wu J, Wang H, et al. Systematic review and network meta-analysis comparing Chinese herbal injections with chemotherapy for treating patients with esophageal cancer. J Int Med Res 2020;48:300060519898336.

(English Language Editor: J. Jones) 